\title{
New Organic Bismuth Hydrangea Materials for Electrochemical Immunoassays Targeting Carcinoembryonic Antigen
}

\author{
Penghui Cao ${ }^{1}$, Xinli Tian ${ }^{1}$, Dong Sun $^{2}$, Zhongmin Wang ${ }^{1}$, Mengkui Ding ${ }^{1}$, Yuhao Li ${ }^{1}$, Xiaoyu Yang ${ }^{1}$, \\ Ruizhuo Ouyang ${ }^{1, *}$, Yuqing Miao ${ }^{1}$ \\ ${ }^{1}$ Institute of Bismuth Science, University of Shanghai for Science and Technology, Shanghai 200093, \\ China \\ ${ }^{2}$ School of Chemistry and Chemical Engineering, Henan Normal University, Xinxiang 453007, China \\ *E-mail: ouyangrz@usst.edu.cn; \\ P. Cao and X. Tian contributed equally to this work.
}

doi: $10.20964 / 2020.10 .06$

Received: 9 January 2020 / Accepted: 23 March 2020 / Published: 31 August 2020

\begin{abstract}
In this work, new organic bismuth submicron hydrangeas (Bi-MEI SMHs) were synthesized for the first time by using 2-methylimidazole (MEI) as a ligand. The material has a porous hydrangea-like structure with large surface area, and exhibits excellent biocompatibility and extremely low toxicity. Bi-MEI SMHs with gold nanoparticles deposited on the outer surfaces were used for antibody capture, thus, providing a new label-free immunosensing platform to quantitatively detect carcinoembryonic antigen (CEA). The performance of the as-developed label free immunosensor was evaluated with different electrochemical techniques. Under optimal conditions, a good linear relationship between the peak current and CEA concentration was observed in a broad range from $1 \mathrm{ng} \mathrm{mL} \mathrm{mL}^{-1}$ to $900 \mathrm{ng} \mathrm{mL}^{-1}$, with a calculated correlation coefficient and detection limit $(\mathrm{S} / \mathrm{N}=3)$ of 0.9880 and $0.3 \mathrm{ng} \mathrm{mL}^{-1}$, respectively. This work provides a new strategy of using organic bismuth materials to fabricate electrochemical immunosensors with promise in sensing platform applications.
\end{abstract}

Keywords: Organic bismuth materials; Bi-MEI; CEA; Electrochemical immunosensor

\section{$\underline{\text { FULL TEXT }}$}

(C) 2020 The Authors. Published by ESG (www.electrochemsci.org). This article is an open access article distributed under the terms and conditions of the Creative Commons Attribution license (http://creativecommons.org/licenses/by/4.0/). 